\title{
O USO DO EXCEL PARA RESOLVER PROBLEMAS DE OPERAÇÕES FINANCEIRAS
}

\author{
Maria Lucia Pozzatti Flores* \\ Mestre em Engenharia de Produção. \\ Professora da Ulbra-SM. Aluna especial do PGIE. \\ mlflores@terra.com.br
}

RESUMO: Com o advento dos microcomputadores a Planilha Eletrônica Microsoft Excel tornou-se uma ferramenta de fácil acesso para professores ou qualquer pessoa que pretenda resolver problemas financeiros. O objetivo deste estudo é apresentar em detalhes as principais funções financeiras da Planilha Eletrônica Excel e mostrar suas aplicações na solução de problemas financeiros, que envolvam quaisquer tipos de fluxos de caixa. Foi desenvolvido um esquema padrão para apresentar a utilização do Excel na solução de problemas envolvendo os parâmetros NPER, TAXA, VP, VF e PGTO, mostrando um número de exemplos em quantidade suficiente para permitir ao usuário desenvolver habilidades suficientes para o seu manuseio.

PALAVRAS-CHAVES: Excel. Operações financeiras.Problemas financeiros.

ABSTRACT: With the use of personal computers, the Microsoft's Excel spreadsheet became an easy access tool for teachers or any other person that needs to solve financials problems using software. The role of this study is to present in details the most important financial functions available at Excel's spreadsheet and show an application that involves any type of cash flow. It was developed a standard routine to show the Excel's use for the solution of problems that involves the parameters NPER, TAXA, VP, VF and PGTO, showing a number of examples that permits users to develop sufficient know-how in their use.

KEYWORDS:Excel. Financial Operation.Financial Problems.

\section{1-INTRODUÇÃO:}

Nos dias de hoje existem mudanças cada vez mais rápidas na área de finanças, e todo profissional deve estar capacitado para administrar com eficiência os recursos disponíveis. Para isso o profissional necessita dominar a matemática financeira, a qual utiliza várias ferramentas para resolver operações financeiros. A utilização da planilha eletrônica é uma destas ferramentas e deve ser de uso comum sempre que uma atividade implique o processamento de um grande volume de cálculos financeiros repetitivos ou simulação de situações envolvendo simultaneamente múltiplas variáveis.

O Microsoft Excel é parte integrante de um software disponível em praticamente todos os computadores e, portanto, acessível para a maioria das pessoas. Mesmo sendo comum nos computadores, nem todas as pessoas o usam; ou por desconhecê-lo ou por trabalharem apenas com parte dele e não com o todo. Além disso, o Microsoft Excel é um programa bastante amigável e portanto de rápida aprendizagem por qualquer pessoa. Desta maneira é uma ferramenta de ensino de fácil acesso a quase todo professor, que poderá utilizá-la para ensinar seus alunos a resolverem operações financeiros.

Nesse estudo vamos usar o Microsoft Excel para resolver problemas financeiros. Inicialmente, apresentaremos como trabalhar na planilha eletrônica Microsoft Excel e os 
requisitos e operadores básicos da matemática elementar. A seguir estudaremos quais são e como trabalhar com as funções financeiras do Microsoft Excel. Finalmente estudaremos como funcionam a capitalização composta, os empréstimos com pagamento unitário ou parcelados e os sistemas de amortização.

\section{2-MATERIAL E MÉTODOS:}

\section{1-Operações Básicas:}

A planilha eletrônica Excel é baseada no conceito de célula, que consiste no elemento matricial definido pela interseção de uma linha com uma coluna. As linhas são numeradas seqüencialmente $(1,2,3,4, \ldots)$ e as colunas por letras em ordem alfabética $(A, B, C, \ldots)$. Por exemplo, a célula B3 é o elemento definido pela intersecção da linha 3 com a coluna B. Quando colocamos o cursor na célula B3, ela fica com seu contorno em negrito, e o número da célula aparece no canto superior da figura. Nessa situação a célula está em condições de receber seu conteúdo mediante sua digitação através do teclado.

Toda a célula que operar com fórmulas deve ter seu conteúdo iniciado pelo sinal de igual(=). Por exemplo: na célula B3 escrevemos $=\mathrm{B} 1+\mathrm{B} 2$, isto significa que B3 será sempre igual à soma dos conteúdos das células B1 e B2.

As funções aritméticas do Excel são realizadas através dos seguintes operadores:

\begin{tabular}{|c|c|}
\hline OPERADOR & DESCRIÇÃO \\
\hline+ & Adição \\
\hline- & Subtração \\
\hline$*$ & Multiplicação \\
\hline$/$ & Divisão \\
\hline$\wedge$ & Exponenciação \\
\hline
\end{tabular}

O sistema Windows é baseado na utilização de menus que permitem ao usuário identificar as funções que devem ser usadas em cada situação. O menu principal do Excel oferece as opções: Arquivo, Editor, Exibir, Inserir, Formatar, Ferramentas, Dados, Janela e Ajuda, colocados nessa ordem, na parte superior da tela do monitor.

\section{2-OPERAÇÕES FINANCEIRAS:}

As convenções adotadas pelo Excel para representar os elementos de um fluxo de caixa são: NPER, TAXA, VP, VF e PGTO, onde:

NPER: número de períodos de capitalização de juros, expresso em anos, semestres, trimestres, meses ou dias.

TAXA: taxa de juros por período de capitalização, expressa em porcentagem, e sempre mencionando a unidade de tempo considerada (ano, semestre,...).

VP: valor presente ou o valor do capital inicial aplicado. Representa o valor monetário colocado no ponto zero.

FV: valor futuro ou o valor do montante colocado no final do período NPER. Representa os valores monetários colocados nas datas futuras $(1,2,3, \ldots)$.

PGTO: valor da prestação da série uniforme que ocorre no final de cada período(postecipada), ou no início de cada período(antecipada).

TYPE: parâmetro para indicar se a convenção adotada é a de final de período $(\mathrm{TYPE}=\mathrm{O})$ ou a de início de período(type=1). 
Em relação aos elementos do Diagrama Padrão:

a)os intervalos de tempo de todos os períodos são iguais.(por exemplo: todos tem a mesma duração igual a 30 dias);

b)os valores do número de períodos NPER podem ser registrados na planilha como números inteiros ou fracionários;

c)a taxa de juros é sempre fornecida em porcentagem;

d)a unidade referencial do tempo deve coincidir com a unidade referencial da taxa;

e) os valores VP, FV e PGTO devem ser registrados com a convenção de sinal. Entradas de caixa sempre positivo; saídas de caixa sempre negativo;

f) convenção de final de período= TYPE 0 (Postecipada); convenção de início de período=TYPE 1 (antecipada). Quando esse parâmetro é omitido assume-se TYPE $=0$.

\subsection{1--ESQUEMA PADRÃO}

Para resolver um problema financeiro devem-se seguir os seguintes passos:

a)na parte inferior da planilha são sempre colocados os valores correspondentes a cada um dos respectivos elementos do fluxo de caixa, que devem ser registrados nas células do Excel;

b)o parâmetro financeiro que não faz parte do problema deve ter seu valor registrado como igual a zero;

c)a célula em destaque é sempre aquela que contém a função financeira do Excel e que provoca o cálculo da operação desejada.

d)o número de períodos de capitalização passa a ser representado na parte superior sempre pela letra n. Quando esse parâmetro for a incógnita devemos inserir a função TAXA;

e)a taxa de juros por período de capitalização passa a ser representada por i. Quando esse parâmetro for a incógnita devemos inserir a função NPER.

\subsection{2-FUNÇÕES FINANCEIRA BÁSICAS}

As funções básicas do Excel que realizam as operações financeiras são:

\begin{tabular}{|c|c|}
\hline $\begin{array}{l}\text { FUNÇÃO } \\
\text { FINANCEIRA }\end{array}$ & PARÂMETROS DA FUNÇÃO \\
\hline PV & (TAXA;NPER;PGTO;VF;TYPE) \\
\hline FV & (TAXA;NPER;PGTO;VP;TYPE) \\
\hline PMT & (TAXA;NPER;VP;VF;TYPE) \\
\hline RATE & (NPER;PGTO;VP;VF;TYPE;ESTIMAÇÃO) \\
\hline NPER & (TAXA;PGTO;VP;VF;TYPE) \\
\hline
\end{tabular}

Os parâmetros de cada função financeira devem ser informados na ordem seqüencial indicados na tabela.

Lembrando que os cinco parâmetros sempre operam nas fórmulas das funções financeiras. Dessa forma, nos problemas com apenas quatro parâmetros, o quinto deve ser necessariamente informado com valor igual a zero.

\section{3-RESULTADOS E DISCUSSÃO:}


A seguir vamos discutir e resolver alguns problemas financeiros, usando somente o Excel:

3.1- Um título é emitido com um prazo de seis meses e com um valor de resgate de $\$ 10.000,00$. Determinar o valor que deve ser aplicado nesse título, na data de emissão, para que a taxa efetiva desse investimento seja igual a $0,85 \%$ ao mês.

Preencher os dados:

$\mathrm{n}=6 \quad \mathrm{i}=0,85 \% \quad \mathrm{PMT}=0,00 \quad \mathrm{FV}=-10.000,00 \quad \mathrm{PV}$

Como o valor de VF foi informado com sinal negativo, VP será obtido com valor positivo.

Com os dados registrados na planilha precisamos colocar a fórmula da função VP, com as operações indicadas a seguir:

-colocar o sinal de igual na célula onde está VP;

-abrir o Inserir no menu principal;

-selecionar a opção Função;

-na janela das funções escolher PV nas funções financeiras. PV (TAXA; NPER; PGTO; VF; TYPE)

Com todos os parâmetros informados, devemos mandar executar a função, e teremos a resposta: $\$ 9.504,84$. Isso quer dizer, que o valor do capital na data da operação é de $\$ 9.504,84$.

3.2- Um financiamento deve ser liquidado através de 6 prestações mensais iguais, com uma taxa de juros compostos de 1,2\% am. Determinar o valor do principal desse financiamento nas seguintes hipóteses:

a)prestação mensal de $\$ 1.700,00$, ocorrendo a $1^{\text {a }}$ prestação um mês após a liberação do principal(série postecipada);

b)prestação mensal de $\$ 1.700,00$, ocorrendo a $1^{\text {a }}$ prestação no ato da liberação do principal(série antecipada).

Preencher os dados:

$\mathrm{n}=6 \quad \mathrm{i}=1,20 \% \quad \mathrm{PMT}=-.1700,00 \quad \mathrm{FV}=0,00 \quad \mathrm{PV}$

a)Série postecipada:TYPE=0

-colocar o sinal de igual na célula PV;

-abrir o Inserir no menu principal;

-selecionar a opção Função;

-na janela das funções escolher VP nas funções financeiras. PV(TAXA; NPER; PGTO;

VF; TYPE). O valor do principal deste financiamento é de $\$ 9.784,95$.

b)Série antecipada: TYPE $=1$.

O resultado é \$9.902,37. Quando a primeira prestação é no ato da operação, o principal será de $\$ 9.902,37$.

3.3- Um título que paga juros anuais foi emitido com quatro cupons anuais de $\$ 800,00$ e com um valor de resgate de $\$ 10.000,00$ no final do $4^{\circ}$ ano. Um investidor pretende comprá-lo na data de emissão e mantê-lo por 4 anos até seu vencimento. Determinar o preço de venda desse título para que esse investidor tenha uma rentabilidade efetiva de $9,5 \%$ aa, assumindo o ano com 360 dias.

Preencher os dados:
$\mathrm{n}=4 \quad \mathrm{i}=9,5 \% \quad \mathrm{PMT}=-800,00$
$\mathrm{FV}=-10.000,00$
PV 
Colocar a fórmula da função VP na célula, seguindo as operações indicadas:

- colocar o sinal de igual na célula;

-abrir o Inserir no menu principal;

-selecionar a opção Função;

-na janela das funções escolher VP nas funções financeiras. PV(TAXA; NPER; PGTO;

VF; TYPE). A função opera como se TYPE=0(postecipada). O preço de venda deste título, na data de emissão é de \$9.519,33.

3.4- Uma instituição financeira recebe uma aplicação de $\$ 10.000,00$, no regime de juros compostos, para ser resgatada num prazo de 9 meses. Determinar o valor de resgate dessa aplicação financeira para que a taxa efetiva de juros seja igual a $10 \%$ aa. Assumir o ano com 360 dias.

Preencher os dados:

$\mathrm{n}=9 / 12=0,75 \quad \mathrm{i}=10 \% \quad \mathrm{PV}=-10.000,00 \quad \mathrm{PMT}=0 \quad \mathrm{FV}$

Colocar a fórmula da função FV, com as operações indicadas:

- colocar o sinal de igual;

-abrir o Inserir no menu principal;

-selecionar a opção Função;

-na janela das funções escolher FV. FV(TAXA;NPER;PGTO;VP;TYPE).

O valor de resgate, 9 meses após a aplicação é de $\$ 10.740,99$.

3.5- Um investidor pretende realizar 10 depósitos mensais de $\$ 5.000,00$, que serão remunerados com a taxa efetiva de $0,90 \%$ am, no regime de juros compostos. Determinar o valor acumulado por esses depósitos, no final de 10 meses, caso o $1^{\circ}$ depósito ocorra no momento em que o investidor decidir pela efetivação dos mesmos.

Preencher os dados:

$\mathrm{N}=10 \quad \mathrm{i}=0,90 \% \quad \mathrm{PV}=0,00 \quad \mathrm{PMT}=-5.000,00 \quad \mathrm{FV}$

Colocar a fórmula da função FV na célula, com as operações:

- colocar o sinal de igual;

-selecionar Inserir no menu principal;

-selecionar a opção Função;

-na janela das funções escolher FV. FV(TAXA;NPER;PGTO;VP;TYPE).

TYPE $=1 \quad$ Portanto, o valor acumulado por esses depósitos é de \$52.543,04.

3.6- Um financiamento de $\$ 10.000,00$ deve ser liquidado em 24 prestações mensais, com a taxa de juros de $0,9 \%$ am, no regime de juros compostos. Determinar o valor da prestação mensal sabendo-se que a $1^{\mathrm{a}}$ prestação ocorre um mês após a liberação dos recursos.

Preencher os dados:

$\mathrm{N}=24 \quad \mathrm{i}=0,90 \% \quad \mathrm{PV}=-10.000,00 \quad \mathrm{FV}=0 \quad \mathrm{PMT}$

Colocar a fórmula da função PMT na célula, com as operações:

-colocar o sinal de igual;

-selecionar Inserir no menu principal;

-selecionar a opção Função;

-na janela das funções escolher PMT. PMT(TAXA;NPER;VP;VF;PGTO)

Portanto, o valor da prestação mensal é de $\$ 465,15$. 
3.7- Um financiamento de $\$ 10.000,00$ deve ser liquidado em 12 prestações mensais de $\$ 880,00$, no regime de juros compostos. Determinar a taxa efetiva de juros desse financiamento sabendo-se que a $1^{\text {a }}$ prestação ocorre um mês após a liberação dos recursos.

Preencher os dados:

$\mathrm{n}=12 \quad \mathrm{PV}=-10.000,00 \quad \mathrm{FV}=0 \quad \mathrm{PMT}=880,00 \quad$ i

Colocar a fórmula da função TAXA na célula, com as operações:

-colocar o sinal de igual;

-selecionar Inserir no menu principal;

-selecionar a opção Função;

-na janela das funções escolher TAXA. TAXA (NPER; PGTO; VP; VF; TYPE;

ESTIMAÇÃO).

A taxa efetiva será de $0,8484004 \%$ am.

3.8- Um financiamento de $\$ 10.000,00$ deve ser liquidado com prestações mensais de $\$ 1.725,48$. Determinar o número de prestações desse financiamento para que a taxa efetiva de juros seja igual a $1,00 \%$ am, no regime de juros compostos. Assumir que a $1^{\mathrm{a}}$ prestação ocorre um mês após a liberação dos recursos.

Preencher os dados:
$\mathrm{i}=1,00 \%$
$\mathrm{VP}=-10.000,00$
$\mathrm{VF}=0$
PGTO $=1.725,48$
$\mathrm{n}$

Colocar a fórmula da função NPER na célula, com as operações:

-colocar o sinal de igual;

-selecionar Inserir no menu principal;

-selecionar a opção Função;

-na janela das funções escolher NPER. NPER(TAXA;PGTO;VP;VF;TYPE).

O número de prestações é de 6,00001314, isto é, 6 meses.

3.9- Um financiamento de $\$ 12.000,00$ deve ser liquidado em 6 anos com a taxa de $8 \%$ aa. Elaborar uma planilha mostrando a prestação, os juros, a amortização e o saldo devedor desta operação.Fazendo pelo método PRICE.

A planilha será:

\begin{tabular}{|c|c|c|c|c|c|}
\hline $1 / \mathrm{A}$ & $\mathrm{B}$ & $\mathrm{C}$ & $\mathrm{D}$ & $\mathrm{E}$ & $\mathrm{F}$ \\
\hline 2 & ANO & $\begin{array}{c}\text { SALDO } \\
\text { DEVEDOR }\end{array}$ & AMORTIZAÇÃO & JUROS & PRESTAÇÃO \\
\hline 3 & 0 & $12.000,00$ & - & - & - \\
\hline 4 & 1 & & & & $2.595,78$ \\
\hline 5 & 2 & & & & \\
\hline 6 & 3 & & & & \\
\hline 7 & 4 & & & & \\
\hline 8 & 5 & & & & \\
\hline 9 & 6 & & & & \\
\hline
\end{tabular}

Em relação aos dados da planilha, temos:

-o valor do principal está registrado na célula C3, que corresponde ao saldo devedor no ano zero. 
-a função PGTO será calculada na célula F4 através das operações:

.colocar o sinal de igual;

.abrir o Inserir no menu principal;

.selecionar a opção Função;

.na janela das funções escolher PGTO. =PGTO $(8 \% ; 6 ;-12.000,00)$. O valor das prestações é de $\$ 2.595,78$.

A planilha continua sendo preenchida na linha correspondente ao $1^{\circ}$ ano:

\begin{tabular}{|c|c|c|c|c|c|}
\hline $1 / \mathrm{A}$ & $\mathrm{B}$ & $\mathrm{C}$ & $\mathrm{D}$ & $\mathrm{E}$ & $\mathrm{F}$ \\
\hline 2 & ANO & $\begin{array}{c}\text { SALDO } \\
\text { DEVEDOR }\end{array}$ & AMORTIZAÇÃO & JUROS & PRESTAÇÃO \\
\hline 3 & 0 & $12.000,00$ & - & - & - \\
\hline 4 & 1 & $10.364,22$ & $1.635,78$ & 960,00 & $2.595,78$ \\
\hline 5 & 2 & & & & \\
\hline 6 & 3 & & & & \\
\hline 7 & 4 & & & & \\
\hline 8 & 5 & & & & \\
\hline 9 & 6 & & & & \\
\hline
\end{tabular}

-O juro do primeiro ano tem valor de $\mathrm{R} \$ 960,00$. A fórmula para o cálculo do juro está colocada na célula $\mathrm{E} 4:=8 \% * \mathrm{C} 3$. A expressão dessa fórmula aparece no visor e o resultado na célula $\mathrm{E} 4$.

-A amortização do $1^{\circ}$ ano tem o valor de $\mathrm{R} \$ 1.635,78$, e está indicada na célula D4, cuja fórmula será: $=\mathrm{F} 4-\mathrm{E} 4$ (prestação do $1^{\circ}$ ano menos os juros do $1^{\circ}$ ano).

Concluída a primeira linha da planilha relativa ao $1^{\circ}$ ano, devemos realizar a operação Preencher/ Para baixo para reproduzir nos anos seguintes as mesmas fórmulas do $1^{\circ}$ ano:

-colocar mouse na célula C4;

-dar clique no botão esquerdo do mouse e mantê-lo pressionado;

-movimentar o mouse para a direta até a célula F4, mantendo o botão esquerdo pressionado;

-com o botão esquerdo ainda pressionado, descer com o mouse, a partir da célula F4, até a célula F9 e soltar o botão do mouse.

Concluídas essas operações, todas as linhas até o $6^{\circ}$ ano estão selecionadas e devemos agora realizar as operações indicadas a seguir:

-abrir Editar no menu principal;

-selecionar a opção Preencher;

-escolher a opção Para baixo.

O resultado está na planilha abaixo:

\begin{tabular}{|c|c|c|c|c|c|}
\hline $1 / \mathrm{A}$ & $\mathrm{B}$ & $\mathrm{C}$ & $\mathrm{D}$ & $\mathrm{E}$ & $\mathrm{F}$ \\
\hline 2 & ANO & $\begin{array}{c}\text { SALDO } \\
\text { DEVEDOR }\end{array}$ & AMORTIZAÇÃO & JUROS & PRESTAÇÃO \\
\hline 3 & 0 & $12.000,00$ & - & - & - \\
\hline 4 & 1 & $10.364,22$ & $1.635,78$ & 960,00 & $2.595,78$ \\
\hline 5 & 2 & $8.597,57$ & $1.766,65$ & 829,14 & $2.595,78$ \\
\hline 6 & 3 & $6.689,59$ & $1.097,98$ & 687,81 & $2.595,78$ \\
\hline 7 & 4 & $4.628,97$ & $2.060,62$ & 535,17 & $2.595,78$ \\
\hline 8 & 5 & $2.403,50$ & $2.225,47$ & 370,32 & $2.595,78$ \\
\hline
\end{tabular}


Com a planilha concluída, vamos mostrar a utilização das funções financeiras PPMT e IPMT, que servem para calcular, respectivamente, os valores dos juros(IPMT) e amortização(PPMT) de uma determinada prestação do modelo Price.

As fórmulas e parâmetros têm a seguinte expressão:

Amortização: = PPMT(TAXA;PER;NPER;VP;VF;TYPE)

Juros: =IPMT(TAXA;PER;NPER;VP;VF;TYPE).

Lembrar que PER corresponde ao número da prestação para a qual se pretende calcular as parcelas de amortização e juros.

No exemplo da planilha acima, determinar os juros e amortização da $5^{\text {a }}$ prestação anual. $\mathrm{PER}=5$

Colocar o sinal de igual em qualquer célula da planilha e abrir Inserir no menu principal, escolher Função e executar essas duas funções financeiras, com os parâmetros a seguir:

$=\operatorname{PPMT}(8 \% ; 5 ; 6 ;-12000 ; 0 ; 0)$ (amortização);

$=\operatorname{IPMT}(8 \% ; 5 ; 6 ;-12000 ; 0 ; 0)$ (juros).

A função PPMT fornece o resultado: $\$ 2.225,47$ para a amortização do $5^{\circ}$ ano; e a função IPMT fornece o valor $\$ 370,32$ para os juros do $5^{\circ}$ ano.

3.10- Um financiamento de $\$ 12.000,00$ deve ser liquidado em 6 anos com a taxa de 8\%aa. Elaborar uma planilha mostrando a prestação, os juros, a amortização e o saldo devedor desta operação.Fazer esse problema pelo método da Amortização Constante.

A planilha será:

\begin{tabular}{|c|c|c|c|c|c|}
\hline $1 / \mathrm{A}$ & $\mathrm{B}$ & $\mathrm{C}$ & $\mathrm{D}$ & $\mathrm{E}$ & $\mathrm{F}$ \\
\hline 2 & ANO & $\begin{array}{c}\text { SALDO } \\
\text { DEVEDOR }\end{array}$ & AMORTIZAÇÃO & JUROS & PRESTAÇÃO \\
\hline 3 & 0 & $12.000,00$ & - & - & - \\
\hline 4 & 1 & & $2.000,00$ & & \\
\hline 5 & 2 & & & & \\
\hline 6 & 3 & & & & \\
\hline 7 & 4 & & & & \\
\hline 8 & 5 & & & & \\
\hline 9 & 6 & & & & \\
\hline
\end{tabular}

Em relação aos dados dessa planilha, temos:

-o valor do principal está registrado na célula C3, que corresponde ao saldo devedor no ano zero.

-o valor da amortização do $1^{\circ}$ ano, colocado na célula $\mathrm{D} 4$, foi obtido pela divisão do principal $(12.000,00)$ pelo prazo do financiamento( 6 anos $).=12000 / 6$

A planilha continua preenchendo a linha correspondente do $1^{\circ}$ ano:

\begin{tabular}{|c|c|c|c|c|c|}
\hline $1 / \mathrm{A}$ & $\mathrm{B}$ & $\mathrm{C}$ & $\mathrm{D}$ & $\mathrm{E}$ & $\mathrm{F}$ \\
\hline 2 & ANO & $\begin{array}{c}\text { SALDO } \\
\text { DEVEDOR }\end{array}$ & AMORTIZAÇÃO & JUROS & PRESTAÇÃO \\
\hline 3 & 0 & $12.000,00$ & - & - & - \\
\hline 4 & 1 & $10.000,00$ & $2.000,00$ & 960,00 & $2.960,00$ \\
\hline
\end{tabular}




\begin{tabular}{|l|l|l|l|l|l|}
\hline 5 & 2 & & & & \\
\hline 6 & 3 & & & & \\
\hline 7 & 4 & & & & \\
\hline 8 & 5 & & & & \\
\hline 9 & 6 & & & & \\
\hline
\end{tabular}

-A fórmula para o cálculo dos juros do $1^{\circ}$ ano está colocada na célula $\mathrm{E} 4:=8 \% * \mathrm{C} 3$. A expressão dessa fórmula aparece no visor e o resultado na célula E4.

-A prestação do $1^{\circ}$ ano tem o valor de $\$ 2.960,00$, e está indicada na célula $\mathrm{F} 4$, cuja fórmula será: = D4+E4 (amortização do $1^{\circ}$ ano mais os juros do $1^{\circ}$ ano).

Concluída a primeira linha da planilha relativa ao $1^{\circ}$ ano, devemos realizar a operação Preencher/ Para baixo para reproduzir nos anos seguintes as mesmas fórmulas do $1^{\circ}$ ano:

-colocar mouse na célula C4;

-dar clique no botão esquerdo do mouse e mantê-lo pressionado;

-movimentar o mouse para a direta até a célula F4, mantendo o botão esquerdo pressionado;

-com o botão esquerdo ainda pressionado, descer com o mouse, a partir da célula F4, até a célula F9 e soltar o botão do mouse.

Concluídas essas operações, todas as linhas até o $6^{\circ}$ ano estão selecionadas e devemos agora realizar as operações indicadas a seguir:

-abrir Editar no menu principal;

-selecionar a opção Preencher;

-escolher a opção Para baixo.

O resultado está na planilha abaixo:

\begin{tabular}{|c|c|c|c|c|c|}
\hline $1 / \mathrm{A}$ & $\mathrm{B}$ & $\mathrm{C}$ & $\mathrm{D}$ & $\mathrm{E}$ & $\mathrm{F}$ \\
\hline 2 & ANO & $\begin{array}{c}\text { SALDO } \\
\text { DEVEDOR }\end{array}$ & AMORTIZAÇÃO & JUROS & PRESTAÇÃO \\
\hline 3 & 0 & $12.000,00$ & - & - & - \\
\hline 4 & 1 & $10.000,00$ & $2.000,00$ & 960,00 & $2.960,00$ \\
\hline 5 & 2 & $8.000,00$ & $2.000,00$ & 800,00 & $2.800,00$ \\
\hline 6 & 3 & $6.000,00$ & $2.000,00$ & 640,00 & $2.640,00$ \\
\hline 7 & 4 & $4.000,00$ & $2.000,00$ & 480,00 & $2.480,00$ \\
\hline 8 & 5 & $2.000,00$ & $2.000,00$ & 320,00 & $2.320,00$ \\
\hline 9 & 6 & 0,00 & $2.000,00$ & 160,00 & $2.160,00$ \\
\hline
\end{tabular}

\section{4-CONCLUSÕES:}

Neste texto deu-se ênfase ao uso do Excel para resolver problemas financeiros. Acredita-se que esse programa possa ser usado com maior freqüência, pois é acessível a qualquer usuário de computador. Sabedor disso o professor de Matemática Financeira deve usá-lo mais em suas aulas como um instrumento a mais para resolver problemas financeiros. Ainda, as funções financeiras básicas são uma ferramenta que facilita a resolução de problemas de juros compostos. O uso da planilha também é bastante simples quando resolvemos problemas de amortização de alguma dívida.

A resolução de problemas financeiros através do uso do Excel é uma alternativa metodológica para o estudo da Matemática Financeira. 


\section{5- REFERÊNCIAS BIBLIOGRÁFICAS:}

-BRUNI,A. L. e FAMÁ, R. Matemática Financeira com HP12C e Excel. 2.ed. São Paulo:Atlas, 2003.

SHINODA,C. Matemática Financeira para usuários do Excel. 2.ed. São Paulo:Atlas, 1998.

LAPPONI,J.C. Matemática Financeira usando Excel. São Paulo:Lapponi Treinamento e Editora Ltda, 1996. 